\title{
Resultados de intervención en un caso pediátrico de Hematocolpos - Piocolpos
}

\author{
Results of intervention in a pediatric case of Hematocolpos - Piocolpos
}

\author{
Aída Isabel Jordán Bolaños ${ }^{1}$, Ruth Aurelia Mejia Ortiz. ${ }^{2}$ \& Sergio Teodomiro Barreno \\ Sanchez. ${ }^{3}$
}

Recibido: 15-01-2020 / Revisado: 20-03-2020 /Aceptado: 14-04-2020/ Publicado: 05-06-2020

\section{DOI: https://doi.org/10.33262/anatomiadigital.v3i2.1..1296}

\begin{abstract}
.
Hematocolpos-piocolpos is one of the very rare pathologies in the Andean region of the equator, generally it follows the pathologies on the alterations in the genesis of the female genitourinary system. With the objective of analyzing the fundamental characteristics in the treatment of a case with hematocolpospiocolpos. A clinical study corresponding to a 9-year-old and 6-month-old pediatric patient was performed. The case was characterized as being prepubertal with a history of abdominal pain, accompanied by a greenish, fetid vaginal discharge. On physical examination, she presented an imperforate
\end{abstract}

\section{Resumen.}

El hematocolpos-piocolpos es una de las patologías poco comunes en la región andina del Ecuador, que ocurre en la génesis del aparato genitourinario de la mujer. Con el objetivo de analizar las características fundamentales en el tratamiento de esta patología, se realizó un estudio clínico de una paciente de 9 años 6 meses de edad. Se trata de una prepúber con antecedentes de dolor abdominal, acompañándose de secreción vaginal verdosa, fétida, al examen físico presentaba himen imperforado. En la ecografía pélvica, fue reportado hematocolpos. La intervención contó con

\footnotetext{
${ }^{1}$ Médico de posgrado de la Especialización Medicina Familiar y Comunitaria II Cohorte Universidad Técnica de Ambato, Ambato, Ecuador, ajordan1281@uta.edu.ec iD https://orcid.org/0000-0002-5131-8495

${ }^{2}$ Médico especialista en Pediatría del Hospital General Ambato IESS. Docente de Pediatría, Universidad Técnica de Ambato. Ambato, Ecuador. ruthmejia1309@yahoo.com iD https://orcid.org/0000-0002-0420-9165

${ }^{3}$ Médico de posgrado de la Especialización Medicina Familiar y Comunitaria II Cohorte Universidad Técnica de Ambato. Ambato, Ecuador. stbarreno1977@gmail.com iD https://orcid.org/0000-0001-8496-1076
} 
hymen. On pelvic ultrasound, hematocolpos were observed. The intervention had 6 medical professionals, all with more than 10 years of experience in gynecological studies. The case had as fundamental results a clinical treatment with antibiotic and surgical treatment, performing hymenectomy plus drainage of purulent material, with a definitive diagnosis of pyometrocolpos, which were sent for culture, with a positive result for Echericha Coli.

Keywords: Hematocolpos-piocolpos, Escherichia Coli, Hymenectomy, Vaginal secretion. especialistas médicos con más de 10 años de experiencia en estudios de ginecología. El tratamiento realizado fue clínico, con antibioticoterapia y quirúrgico, en el que se realizó himenectomia más drenaje de material purulento; el diagnóstico definitivo fue de piometrocolpos. Se enviaron las muestras para cultivo, reportándose positivo para Echericha Coli.

\section{Introducción.}

Palabras claves: Hematocolpos-piocolpos, Echericha Coli, Himenectomia, Secreción vaginal.

Durante la formación del desarrollo humano, muchas son las anomalías que se pueden presentar en el aparato reproductor femenino.(Vázquez-Pizaña et al., 2006). Estas se manifiestan en cualquier momento del desarrollo, pero su mayor parte al nacimiento. Las malformaciones en muchas ocasiones no son descubiertas al nacer, por lo que la mayoría, no tiene un diagnóstico previo y son identificadas solo tiempo después.

Los trastornos congénitos del tracto genital femenino son poco frecuentes, su incidencia es de 1:10000-20000, recién nacidas vivas (Mar1 et al., 2016). Pueden presentarse con múltiples manifestaciones clínicas, que en ocasiones son graves, por lo que es necesario hacer un diagnóstico rápido y oportuno (Paul et al., 2019).

Según diferentes autores, (Banale et al., 2013), (Birge et al., 2015), (Park et al., 1999) la existencia de piocolpos se produce debido a una infección del flujo vaginal, este es un cuadro muy poco frecuente y su incidencia se estima en un 0,1 a 0,05\%. En el Hospital General Ambato desde el año 2010 hasta la actualidad solo se han presentado un total de 6 casos, lo que representa el $0,6 \%$ de este padecimiento por año calendario.

Según (Józwik \& Józwik, 2000), la vía de infección del flujo retenido que se presenta con mayor frecuencia es la hematógena; de ahí la importancia de su drenaje sin demora. No se puede descartar que el piometrocolpos ha ido creciendo en los últimos años y es muy común asociarlo a infecciones urinarias, retención de líquidos vaginales y gérmenes poco frecuentes, 
debido al aplastamiento que el piocolpos origina sobre los uréteres; Incluso puede llegar a originar cuadros severos de hidronefrosis y abscesos renales (Garba et al., 2018). Para cada uno de estos casos, el correcto diagnóstico, la construcción de una buena historia clínica, una eficiente exploración física y las pruebas de imágenes constituye la base de un buen diagnóstico. (Patel et al., 2017). La ecografía abdominal puede mostrar una tumoración que puede llegar a ocupar todo el hipogastrio e hipocondrio, con contenido ecodenso en su interior. En ocasiones, el piocolpos puede ser de tal cuantía que origine piometra e incluso piosálpinx. La resonancia magnética es útil para el diagnóstico diferencial de otras lesiones ocupantes (Birge et al., 2015) Actualmente con el avance de la tecnología podemos realizar un diagnóstico ecográfico prenatal, especialmente cuando se sospecha de mucocolpos intraútero (Duyos Mateo et al., 2012).

El hematocolpos es una malformación congénita rara, causada por una alteración en los conductos de Müller y mesonéfricos que se caracteriza por la triada: útero didelfo, hemivagina obstruida y agenesia renal ipsilateral, aún de etiología incierta. Las pacientes suelen permanecer asintomáticas hasta la menarquia. El síndrome de hemivagina obstruida es excepcional y ocurre como consecuencia de una alteración embriológica en la octava semana de gestación.(Paul et al., 2019)

Para el caso del hematocolpos- piocolpos, es obligatorio tener un conocimiento prematuro de su diagnóstico en toda adolescente en la etapa de desarrollo puberal, cuando presentan amenorrea primaria (I. Delgado Pecellín, V.M. Navas López A. Rodríguez Herrera, B. Espín Jaime, 2007) y antes que se visualice una masa abdominal a nivel de hipogastrio. (Duyos Mateo et al., 2012).

\section{Presentación del caso clínico}

Paciente de 9 años 6 meses, sin antecedentes personales, ni familiares de importancia, que acude al servicio de emergencia del Hospital General Docente Ambato, por dolor abdominal localizado en hipogastrio y secreción vaginal amarillenta escasa, de un mes de evolución, tratada con cefalosporinas de primera generación, sin mejoría de la sintomatología. Doce horas antes del ingreso presenta exacerbación de dicho dolor localizado en el mismo lugar, fiebre no cuantificada y aumento de secreción verdosa por vagina, de mal olor, en abundante cantidad. Al examen físico, los signos vitales estaban dentro de la normalidad para la edad, con aparente buen estado general, lúcida, bien orientada. Cardiopulmonar: normal. El abdomen se presentaba doloroso a la palpación en el hipogastrio, sin signos de irritación peritoneal, a nivel de genitales se observó, abombamiento del himen con salida de secreción purulenta fétida en abundante cantidad, por un orificio himeneal mínimo en hora 9, con eritema vulvar. Se realizó una ecografía pélvica, encontrándose colección liquida en vagina, con aspecto de hematoma, con un volumen de $53 \mathrm{ml}$, con diagnostico presuntivo de hematocolpos. Figura 1. 


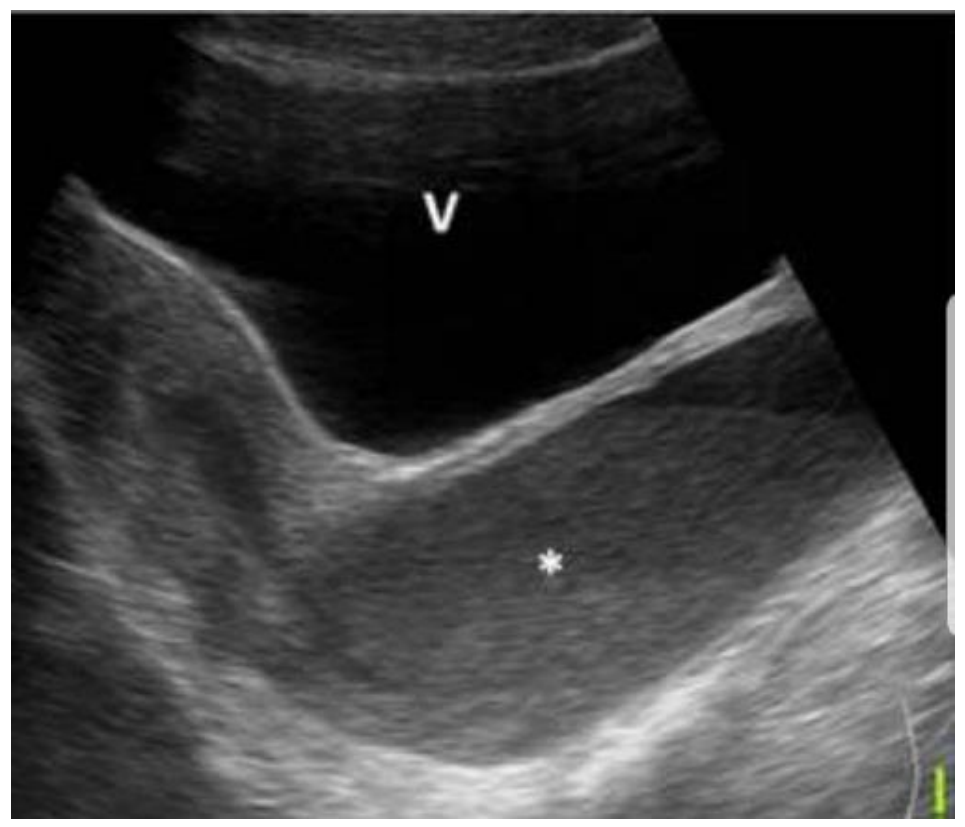

Figura 1. Colección liquida en vagina, con aspecto de hematoma, volumen de $53 \mathrm{ml}$ Fuente: Elaboración propia.

Para el cuidado de los aspectos éticos del presente estudio, se solicitó el consentimiento informado por parte de la paciente y su madre. Nuestro objetivo fue presentar un material completo y con sustento científico teórico y visual. La paciente y su madre no presentaron restricciones de ningún tipo para la presentación de las imágenes del caso.

\section{Resultados}

En cuanto al tratamiento quirúrgico, se realizo una himenectomia (Figura 2), visualizándose la mucosa vaginal eritematosa con cérvix de aspecto verdoso y presencia de material purulento, procediéndose al drenaje del mismo; en el proceso, se administró antibioticoterapia intravenosa con Cefotaxima, Gentamicina y Metronidazol, observándose por parte de la paciente una mejora clínica. Al cuarto día de su estancia hospitalaria es dada de alta en buenas condiciones generales. Los restos de material extraídos fueron enviados para la realización del cultivo, cuyos resultados fueron positivos para Echericha Coli. Figura 3.

Se realizó posteriormente una visita domiciliar, por parte del grupo de seguimiento médico, encontrándose a la paciente asintomática y en el examen físico de la región genital se observó el orificio himeneal permeable y sin hallazgos negativos. 
Figura 2. Himenectomia realizada por parte del cuerpo investigativo Fuente: Elaboración propia.

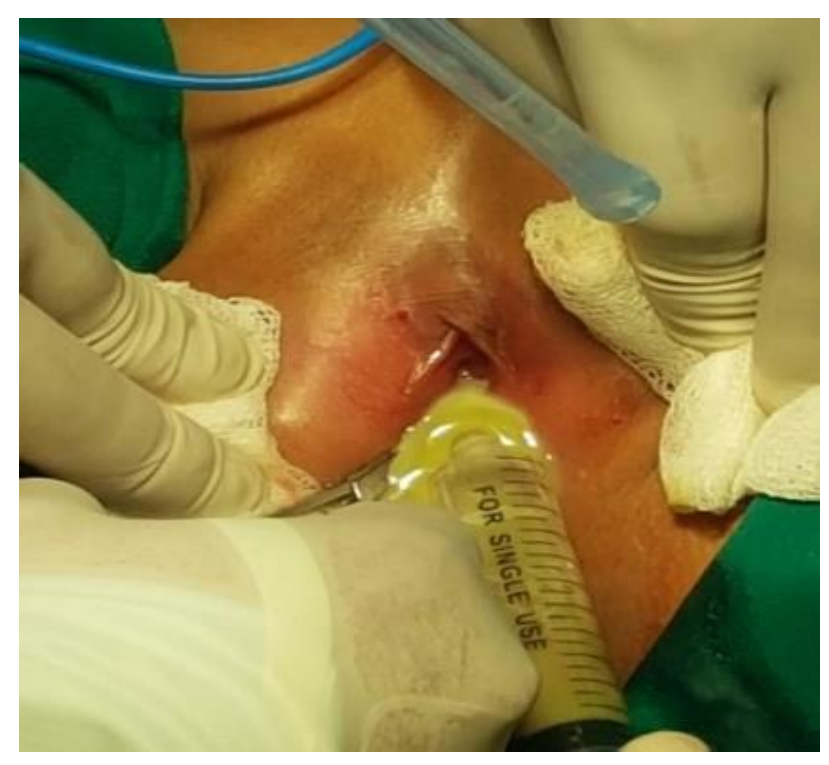

Figura 3. Muestra enviada para estudio histopatológico Fuente: Elaboración propia.

\section{Discusión}

El Hematocolpos-piocolpos, es una patología rara en nuestro medio, que se produce por una malformación congénita, causada por una alteración en el desarrollo a nivel de los conductos de Müller y mesonéfricos, muchas de las veces permanece asintomático durante la infancia, o al contrario pueden presentar una masa a nivel de hipogastrio, dolor abdominal o lumbar, molestias o infecciones urinarias, amenorrea, hematocolpos o hematómetra, estas últimas en pacientes adolescentes. La etiología más frecuente puede ser el himen imperforado, septum vaginal tranverso, seno urogenital y malformación cloacal. En otras ocasiones el diagnóstico es un hallazgo casual al realizarse una ecografía. Nuestra paciente presento dolor abdominal, acompañándose posteriormente de salida de secreción purulenta, cuya causa fue himen imperforado, que fue de resolución quirúrgica.

En la literatura hemos encontrado varios estudios clínicos, como, Manejo quirúrgico de un caso de hematocolpos por tabique vaginal transverso (Jorge Gutarra Canchucaja, Imelda Leyton Valencia, Francisco Esteves Osso, Milthon Mestanza Sáenz, 2013) A través del orificio se pasó una sonda Foley de triple vía № 16, se insufló el globo con 30 ml de suero 
fisiológico, exponiendole al tabique mediante tracción, procediéndose a cortarle en rodete alrededor de la sonda con electrobisturí, en un radio de $6 \mathrm{~mm}$. A pesar de que no está estrechamente relacionado con el caso anteriormente presentado, sirvió de base para conocer las diferentes formas de actuación en casos clínicos similares.

En otro estudio, Hematocolpos en la infancia. A propósito de un caso (Espinosa Gómez et al., s. f.), fue realizado una intervención quirúrgica electiva bajo anestesia general. Se hizo una incisión y resección de la porción central de la membrana himeneal y el drenaje de 500 $\mathrm{ml}$ de sangre acumulada. Se colocó férula de goma, para mantener permeable el canal vaginal, retirándose siete días después, observándose en la paciente evolución satisfactoria. En nuestra paciente, el diagnóstico fue Hematocolpos-piocolpos, cuya etiología fue himen imperforado y el tratamiento quirúrgico fue himenectomia, similar a los estudios de casos reportados con drenaje del contenido purulento, luego de lo cual la paciente presento una evolución favorable.

\section{Conclusiones}

Los autores mediante este estudio de caso queremos llamar la atención del personal médico general, neonatologos, sobre un completo y adecuado examen físico en el recién nacido, en las primeras horas de vida, para identificar alteraciones diversas. Señalándoles la importancia de la prevención, ya que nos evitaríamos a futuro, patologías infecciosas a nivel del sistema genital femenino con sus complicaciones que pueden llegar a ser graves y comprometer la vida de las niñas o adolescentes.

Además el Hematocolpos-Piocolpos es una condición muy poco frecuente en la vida de las féminas en edades pediátricas, sin embargo, debe considerarse dentro de las posibilidades diagnósticas. Su tratamiento por ser sencillo no deja de ser riguroso y de extremo cuidado, ya que las intervenciones quirúrgicas pueden complicarse, debiendo realizarse una nueva intervención.

\section{Referencias bibliográficas}

Banale, K., Javali, T., Babu, P., Jyothi, G. S., Shetty, P., Nagaraj, H. K., \& Gaurishankar, S. (2013). Fetus in bladder. Indian journal of urology: IJU : journal of the Urological Society of India, 29(4), 351-352. https://doi.org/10.4103/0970-1591.120123

Birge, O., Ozbey, E. G., Erkan, M. M., Arslan, D., \& Kayar, I. (2015). Youssef’s Syndrome following Cesarean Section. Case reports in obstetrics and gynecology, 2015, 605325. https://doi.org/10.1155/2015/605325

Duyos Mateo, I., Abehsera Davó, D., de la Calle Fernández-Miranda, M., Puch Botella, M., 
\& del Mar González Arlanzón, M. (2012). El himen imperforado como urgencia en ginecología: Caso clínico y revisión de la literatura. Progresos de Obstetricia y Ginecologia, 55(9), 445-448. https://doi.org/10.1016/j.pog.2012.05.005

Espinosa Gómez, I., Ponce Sandoval, N., Manuel González Valdés, V., Mora Casacó, B., González Ricardo, Y., \& del Carmen Marco Maya, M. (s. f.). Hematocolpos en la infancia. A propósito de un caso Hematocolpos in Childhood. A case Report.

Garba, I., Rabiu, A., Ahmed, Z., \& Ozegya, M. (2018). Vesicouterine fistula (Youssef's Syndrome): A case report and review of literature. Nigerian Journal of Basic and Clinical Sciences, 15(1), 101. https://doi.org/10.4103/njbcs.njbcs_43_17

I. Delgado Pecellín, V.M. Navas López A. Rodríguez Herrera, B. Espín Jaime, A. P. M. y J. R. R. R. (2007). Himen imperforado como causa infrecuente de dolor abdominal recurrente. Anales de Pediatría. https://www.analesdepediatria.org/es-pdf-13107402

Jorge Gutarra Canchucaja, Imelda Leyton Valencia, Francisco Esteves Osso, Milthon Mestanza Sáenz, L. C. (2013). Manejo quirúrgico de un caso de hematocolpos por tabique vaginal transverso. Revista Peruana de Ginecología y Obstetricia. http://www.scielo.org.pe/scielo.php?script=sci_arttext\&pid=S230451322013000200009

Józwik, M., \& Józwik, M. (2000). Clinical classification of vesicouterine fistula. International Journal of Gynecology \& Obstetrics, 70(3), 353-357. https://doi.org/10.1016/S0020-7292(00)00247-2

Marı, A., Serrano, C., Lo, E., \& Gonza, G. (2016). Obstetricia Y Ginecolog '. 58(8), 368372.

Park, B. K., Kim, S. H., Cho, J. Y., Sim, J. S., \& Seong, C. K. (1999). Vesicouterine fistula after cesarean section: Ultrasonographic findings in two cases. Journal of Ultrasound in Medicine, 18(6), 441-443. https://doi.org/10.7863/jum.1999.18.6.441

Patel, A. R., Patra, F., Shah, N. P., \& Shukla, D. (2017). Biological control of mycotoxins by probiotic lactic acid bacteria. Dynamism in dairy industry and consumer demands, 2015(February), 2-4. https://doi.org/10.1155/2015

Paul, J., Osuna, N., Alberto, E., Ramírez, L., Iván, F., \& Amador, G. (2019). Anomalías del conducto mülleriano : Síndrome de Herlyn-Werner-Wunderlich a pro-pósito de un caso Mullerian conduct anomalies : Herlyn-Werner-Wunderlich syndrome a case report. 9(1), 33-37.

Vázquez-pizaña, E., To, A., Rojo-quiñónez, R., Er Ic Var, O., To Gómez-river, N., Mo López-cer, G., \& Pediatra, M. (2006). Malfor maciones del Apar ato Genital Femenino en la Adolescencia. En Bol Clin Hosp Infant Edo Son (Vol. 23, Número 2).

von Carolina Garfias, F., Antonio Barrera, N., Ricardo Zubieta, A., \& Francisca Ugarte, P. (2011). Neonatal hydrocolpos and hydrometrocolpos: A clinical case. Revista Chilena de Pediatria, 82(2), 137-141. https://doi.org/10.4067/S0370-41062011000200008 
Vol. 3, N².1, p. 51-58, junio, 2020

PARA CITAR EL ARTÍCULO INDEXADO.

Jordán Bolaños , A. I., Mejia Ortiz, R. A., \& Barreno Sanchez, S. T. (2020). Resultados de intervención en un caso pediátrico de Hematocolpos - Piocolpos. Anatomía Digital, 3(2.1.), 51 58. https://doi.org/10.33262/anatomiadigital.v3i2.1.1296

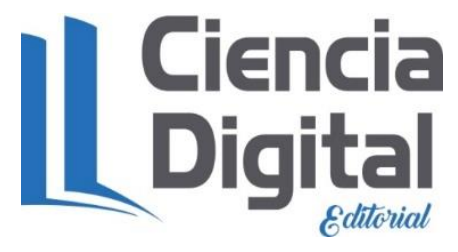

El artículo que se publica es de exclusiva responsabilidad de los autores y no necesariamente reflejan el pensamiento de la Revista Anatomía Digital.

El artículo queda en propiedad de la revista y, por tanto, su publicación parcial y/o total en otro medio tiene que ser autorizado por el director de la Revista Anatomía Digital.
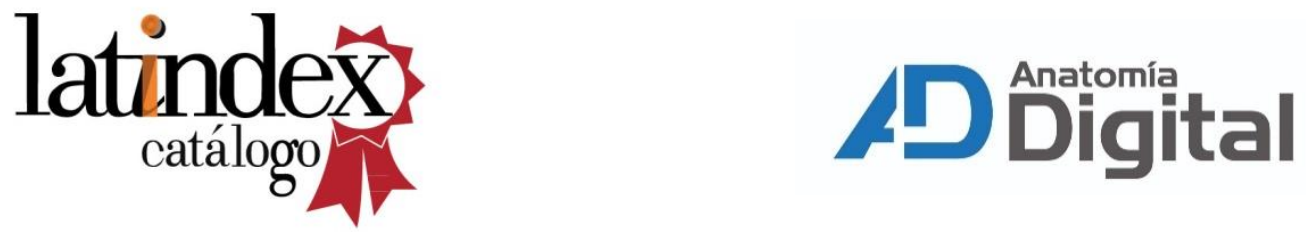